\title{
Residual high incidence of ventricular arrhythmias after left ventricular reconstructive surgery
}

James 0. O'Neill, MB, FRCPI, ${ }^{a}$ Randall C. Starling, MD, MPH, FACC, ${ }^{a}$ Yaariv Khaykin, MD, ${ }^{\mathrm{b}}$ Patrick M. McCarthy, MD, James B. Young, MD, MPH, FACC, ${ }^{a}$ Melanie Hail, RN, ${ }^{d}$ Nancy M. Albert, PhD, RN, ${ }^{d}$ Nicholas Smedira, MD, ${ }^{\mathrm{c}}$ and Mina K. Chung, MD, FACC ${ }^{b}$

From the Department of Cardiovascular Medicine, Section of Heart Failure and Cardiac Transplant Medicine, ${ }^{\text {a }}$ Department of Cardiovascular Medicine, Section of Cardiac Electrophysiology and Pacing, ${ }^{\text {b }}$ Department of Cardiothoracic Surgery, ${ }^{\mathrm{c}}$ and Department of Cardiovascular Medicine, Kaufman Center for Heart Failure, ${ }^{d}$ The Cleveland Clinic Foundation, Cleveland, Ohio.

Dr O'Neill receives support from the Fulbright Commission.

Received for publication Feb 17, 2005; revisions received June 27, 2005; accepted for publication June 30, 2005

Address for reprints: Mina K. Chung, MD, FACC, Department of Cardiovascular Medicine, Cleveland Clinic Foundation, Desk F15, 9500 Euclid Ave, Cleveland, OH 44195 (E-mail: Chungm@ccf.org).

J Thorac Cardiovasc Surg 2005;130:1250-6

$0022-5223 / \$ 30.00$

Copyright (C) 2005 by The American Association for Thoracic Surgery

doi:10.1016/j.jtcvs.2005.06.045
Objective: Left ventricular reconstruction is performed in patients with ischemic cardiomyopathy and akinetic or dyskinetic left ventricular regions. These patients may remain at risk for malignant ventricular arrhythmias and hence may benefit from prophylactic implantable cardioverter-defibrillators. Specific guidelines for electrophysiologic testing and implantable cardioverter-defibrillator implantation in patients undergoing left ventricular reconstruction are lacking. We aimed to assess the residual risk and timing of ventricular arrhythmias after left ventricular reconstruction to determine whether electrophysiologic risk stratification or implantable cardioverter-defibrillator implantation can be safely deferred.

Methods: Data were prospectively gathered on 217 consecutive patients with left ventricular ejection fractions less than $40 \%$ undergoing left ventricular reconstruction at our institution from 1997 to 2002. Patients were divided into 3 groups: group 1, implantable cardioverter-defibrillator present before surgery; group 2, implantable cardioverter-defibrillator implanted early after surgery; and group 3, no implantable cardioverter-defibrillator implanted. End points were all-cause mortality (censored for cardiac transplantation) and appropriate implantable cardioverter-defibrillator therapies.

Results: Of 217 patients (mean age, $61 \pm 10$ years [mean $\pm \mathrm{SD}$ ]), survival after a median follow-up of 381 days was $90 \%$. Electrophysiologic studies successfully identified patients at low risk. Appropriate implantable cardioverter-defibrillator therapies occurred in $20 \%$ of group 1 and $12 \%$ of group 2. The median time to the first implantable cardioverter-defibrillator therapy from the time of left ventricular reconstruction was 43 days, and most first therapies (67\%) occurred within the first 63 days.

Conclusions: The early event rates (occurring in the first 90 days after left ventricular reconstruction) support the use of predischarge electrophysiologic studies, implantation of implantable cardioverter-defibrillators before discharge from the hospital, or both.

$\mathrm{L}$ eft ventricular reconstruction (LVR), or the modified Dor procedure, has been performed with increasing frequency in patients with severe ischemic heart disease, ventricular dysfunction, and heart failure. However, its longterm efficacy remains to be established and is the subject of the STICH (Surgical Treatments for IsChemic Heart Failure) trial, which will compare medical therapy with coronary artery bypass grafting (CABG) alone and CABG in addition to LVR surgery. ${ }^{1}$ Although these approaches are intended to reduce mortality due to coronary ischemia or heart failure, patients may remain at risk for mortality due to life-threatening arrhythmias.

Patients who have undergone recent cardiac operations have been excluded in some of the major trials examining the benefit of early arrhythmia risk stratification, implantable cardioverter-defibrillator (ICD) therapy, or both. The Multicenter Un- 


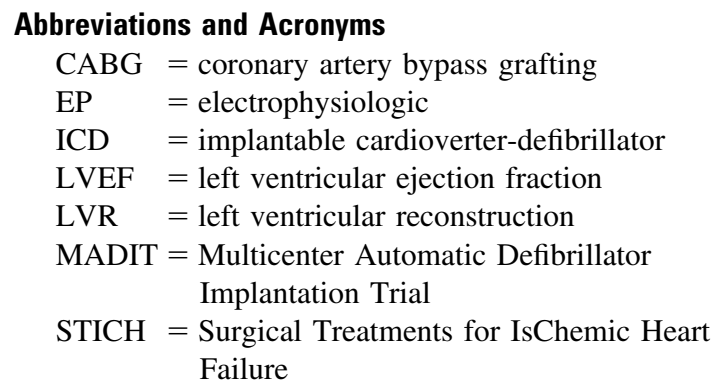

sustained Tachycardia Trial, which reported survival benefits for ICD implantation in ischemic cardiomyopathy with nonsustained ventricular tachycardia and inducible sustained ventricular arrhythmias, did include patients 4 days after surgery. However, the Multicenter Automatic Defibrillator Implantation Trial (MADIT), which reported that ICD therapy improved survival in patients with prior myocardial infarction and left ventricular dysfunction, excluded patients within 2 months (MADIT) or 3 months (MADIT II) after CABG. ${ }^{2,3}$ Furthermore, the Centers for Medicare \& Medicaid Services state that an ICD is reasonable and necessary in patients with a left ventricular ejection fraction (LVEF) less than $30 \%$, but only after 3 months have elapsed since operation. ${ }^{4}$ There are no clear guidelines regarding the necessity of early ICD implantation in patients undergoing LVR.

We used a customized, prospective database to assess (1) the risk of ventricular arrhythmias after LVR and (2) the timing of events after LVR to determine whether electrophysiologic (EP) risk stratification or ICD implantation are warranted before hospital discharge.

\section{Methods}

\section{Patient Population}

Patients were selected for LVR as part of a comprehensive nontransplantation surgical approach to their heart failure syndrome. ${ }^{5}$ In practice, patients were assessed by cardiac magnetic resonance imaging and 2- and 3-dimensional echocardiography in addition to left ventriculography and coronary angiography. Consideration for LVR was undertaken in patients whose myocardial scar was confined to a single coronary territory (almost always the left anterior descending artery). The final decision to perform LVR was made by the surgeon at the time of operation. Data were prospectively recorded in a customized database on consecutive patients undergoing LVR in our institution from 1997 to 2002. The database was approved by the Institutional Review Board of the Cleveland Clinic Foundation. Patients who survived until hospital discharge and, thus, were eligible for EP testing were included for analysis. The intent was for all patients to undergo EP studies, at the discretion of the treating physician. When they were not performed, it was largely due to patient refusal or logistic reasons. Patients were excluded for any of the following reasons: emer- gency operation, preoperative inotropic dependence, or preoperative LVEF greater than $40 \%$.

\section{Surgical Technique}

The rationale for and description of LVR are described elsewhere ${ }^{6}$; LVR was performed primarily for anterior myocardial scarring. ${ }^{7}$ Briefly, the procedure was performed with cardiopulmonary bypass, and coronary revascularization, where needed, was performed initially, followed by mitral valve annuloplasty through a left atriotomy if there was significant mitral regurgitation. After revascularization and mitral valve repair, LVR was performed. The left ventricle was opened through the apical scar, and palpation with or without intraoperative echocardiography was used to define the border zone between infarcted and normal myocardium. A purse-string polypropylene suture was placed and tied through this border zone to create a neck. Although the original description of the procedure includes an endoventricular Dacron (DuPont, Wilmington, Del) patch to exclude the infarcted myocardium from the left ventricular cavity, in most cases LVR was accomplished by the placement of additional sutures, without a Dacron patch. Cryoablation was performed in $13 \%$ of patients. At the time of operation, the presence of scarring (dyskinesis) or an akinetic region was adjudicated by the surgeon, rather than by imaging modalities.

\section{EP Testing}

EP testing was performed on most patients before discharge, at the discretion of the treating physician. The EP study was performed with patients in the fasting state by using programmed ventricular stimulation. The stimulation protocols consisted of programmed ventricular stimulation with either (1) up to 3 ventricular extrastimuli, after 8-beat paced drive cycles at up to 2 paced cycle lengths at 2 right ventricular endocardial sites, ${ }^{8-11}$ or (2) 4 extrastimuli at 3 paced cycle lengths at 2 right ventricular sites. ${ }^{12}$ Burst pacing, short-long-short coupling intervals, or both were also performed in some patients. The specific stimulation protocol was at the discretion of the physician. A positive study was defined as inducible sustained ventricular tachyarrhythmias (monomorphic or polymorphic) lasting more than 30 seconds or associated with syncope, hemodynamic compromise, or the necessity for intervention for termination. A negative EP study was defined as noninducibility of sustained ventricular tachyarrhythmias. The decision to implant an ICD was made on the basis of late ( $>48$ hours) documented life-threatening ventricular arrhythmias after LVR (secondary prevention of sudden cardiac death) or for a positive EP study (primary prevention).

\section{Follow-up and End Points}

Demographic, echocardiographic, clinical status, and ICD interrogation data were collected. All patients with ICDs underwent regular scheduled device checks in the arrhythmia device clinic. Survival status was obtained from the Social Security Death Index, physician follow-up, review of medical records, or patient contact. End points were all-cause mortality and appropriate ICD therapies. ICD therapies were obtained from device interrogation and adjudicated as being appropriate by an electrophysiologist. When a death occurred, every effort was made to ascertain the cause of death. The World Health Organization definition of sudden cardiac 


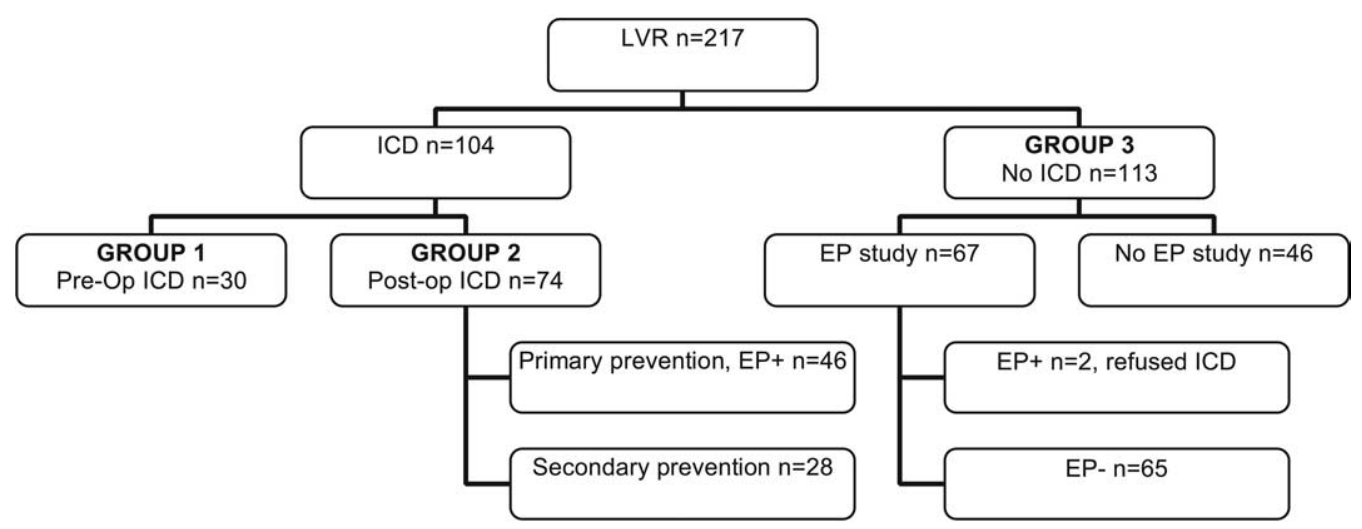

Figure 1. Description of the 3 groups. $L V R$, Left ventricular reconstruction; ICD, implantable cardioverter-defibrillator; $E P$, Electrophysiologic; Pre-op, before surgery; Post-Op, after surgery.

death was used: "an unexpected death due to cardiac problems which occurs within 1 hour from the start of any cardiac related symptoms."

\section{Statistical Analysis}

Patients were divided into 3 groups on the basis of ICD status: group 1 had an ICD in situ before surgery, group 2 had an ICD implanted after surgery, and group 3 had no ICD implanted (Figure 1). Survival is presented according to the method of Kaplan and Meier. For survival analysis, patients who underwent orthotopic cardiac transplantation were censored at the date of transplantation. Survival curves were compared by the log-rank test. Cox proportional hazards methodology was used to assess the effect of independent variables on survival or ICD discharge. The KruskalWallis test was used for comparisons of continuous variables, and the $\chi^{2}$ test was used to test for comparisons of categorical variables. Unless stated otherwise, data are expressed as mean $\pm \mathrm{SD}$.

\section{Results}

Patient characteristics are shown in Table 1. Of 219 patients undergoing LVR, 217 survived to hospital discharge and were included in the analysis. The mean age of the overall cohort $(\mathrm{n}=217)$ was $61 \pm 10$ years (range, $29-83$ years). Most patients (82\%) were male, $28 \%$ had diabetes, and $48 \%$ had hypertension. Concomitant $\mathrm{CABG}$ was performed in $88 \%, 46 \%$ underwent mitral valve repair, cryoablation was performed in $13 \%$, and a patch was applied in $16 \%$ overall. The types of surgery performed in the 3 groups were similar, with the exception of cryoablation, which was more commonly performed in group 1 as a result of the higher incidence of preoperative ventricular arrhythmias in these patients. At operation, $67 \%$ had dyskinetic scars, and $33 \%$ had akinetic regions as determined by the surgeon. No patient required cardiac transplantation before discharge. During the follow-up period, 4 patients subsequently underwent cardiac transplantation ( 2 from group 1 and 2 from group 2).
Of the 217 patients included in the study, 104 were discharged with an ICD (Figure 1). Thirty patients (group 1) had ICDs in situ before undergoing operation. After LVR, indications for ICD implantation were secondary prevention in 28 patients and primary prevention in 46. For the 28 patients who received an ICD for secondary prevention, the qualifying arrhythmia occurred before surgery in 8 and after surgery in 20. Four patients with negative EP studies had ICD implantation performed because of late ( $>48$ hours) life-threatening ventricular arrhythmias after LVR.

Of the 113 patients who did not receive an ICD, 46 patients did not undergo EP study. The decision of whether to refer a patient for EP study was at the discretion of the treating physician. The intent was for all patients to undergo EP testing. However, patient refusal and logistical issues prevented this on some occasions. Of the patients in groups 2 and 3, 113 patients had an EP study, and 48 (42\%) were positive; 2 patients with positive EP studies refused ICD implantation (Figure 1).

There were significant differences among the 3 groups (Table 1). Patients in group 1 were the sickest cohort, with the highest percentage $(80 \%)$ of dyskinetic scars, the largest end-diastolic dimensions, the most frequent requirement for mitral valve surgery, and the lowest proportion of concomitant revascularizations. Furthermore, group 1 had longer QRS durations on their resting electrocardiograms, and there was a trend for them to be older. Cryoablation was performed more frequently in group 1 than in groups 2 or 3; this is indicative of the incidence of ventricular arrhythmias in patients who had ICDs before undergoing LVR.

The median follow-up was 381 days (range, 6-1601 days). Follow-up was complete in all patients. Annualized mortality was $7.14 \% / y$ in group 1 and was significantly lower in groups $2(0.03 \% / y)$ and $3(0.06 \% / y ; P=.04)$. Overall survival was $90 \%$. Compared with group 1, survival 
TABLE 1. Clinical characteristics, perioperative data, and outcomes for all patients and specified subgroups

\begin{tabular}{|c|c|c|c|c|c|}
\hline \multirow[b]{2}{*}{ Variable } & \multirow{2}{*}{$\begin{array}{c}\text { All } \\
\text { patients }\end{array}$} & \multicolumn{3}{|c|}{ Group } & \multirow{2}{*}{$\begin{array}{c}P \text { value } \\
\text { between } \\
\text { groups }\end{array}$} \\
\hline & & 1 & 2 & 3 & \\
\hline $\mathrm{n}$ & 217 & 30 & 74 & 113 & \\
\hline \multicolumn{6}{|l|}{ Preoperative patient characteristics } \\
\hline$\%$ Male & 82 & 80 & 86 & 80 & .47 \\
\hline Age (y) & $61 \pm 10$ & $62 \pm 9$ & $59 \pm 10$ & $62 \pm 9$ & .11 \\
\hline Hypertension (\%) & 48 & 43 & 45 & 52 & .50 \\
\hline Diabetes (\%) & 28 & 10 & 30 & 31 & .07 \\
\hline Amiodarone $(\%)$ & 39 & 63 & 54 & 23 & .01 \\
\hline LVEF (\%) & $22 \pm 8$ & $18 \pm 8$ & $22 \pm 6$ & $23 \pm 8$ & .01 \\
\hline LVEDD (cm) & $6.4 \pm 0.9$ & $6.8 \pm 0.8$ & $6.4 \pm 0.8$ & $6.3 \pm 0.9$ & .03 \\
\hline LVESD (cm) & $5.1 \pm 1.0$ & $5.7 \pm 1.0$ & $5.2 \pm 0.9$ & $4.9 \pm 1.0$ & .03 \\
\hline QRS (ms) & $123 \pm 32$ & $157 \pm 36$ & $122 \pm 31$ & $114 \pm 25$ & .01 \\
\hline Dyskinetic scar (\%) & 67 & 80 & 68 & 67 & .24 \\
\hline \multicolumn{6}{|l|}{ NYHA class $(\%)$} \\
\hline I & 3 & 0 & 1 & 4 & \\
\hline II & 29 & 13 & 30 & 3 & \\
\hline III & 47 & 43 & 55 & 43 & \\
\hline IV & 21 & 43 & 14 & 20 & .01 \\
\hline \multicolumn{6}{|l|}{ Operative procedure } \\
\hline CABG $(\%)$ & 88 & 77 & 92 & 88 & .10 \\
\hline Mitral valve surgery (\%) & 46 & 63 & 45 & 43 & .14 \\
\hline Patch $(\%)$ & 16 & 17 & 12 & 18 & .59 \\
\hline Cryoablation (\%) & 13 & 57 & 8 & 5 & .01 \\
\hline \multicolumn{6}{|l|}{ End points } \\
\hline Follow-up, d (median \pm IOR) & $381 \pm 660$ & $248 \pm 316$ & $380 \pm 716$ & $525 \pm 671$ & .01 \\
\hline Deaths, n (\%) & $20(9)$ & $6(20)$ & $3(4)$ & $11(10)$ & .04 \\
\hline Transplantation (n) & 4 & 2 & 2 & 0 & .91 \\
\hline ICD therapy, n (\%) & $\mathrm{N} / \mathrm{A}$ & $6(20)$ & $9(12)$ & $\mathrm{N} / \mathrm{A}$ & .38 \\
\hline
\end{tabular}

Data are mean \pm SD unless otherwise noted. $L V E F$, Left ventricular ejection fraction; $L V E D D$, left ventricular end-diastolic dimension; $L V E S D$, left ventricular end-systolic dimension; NYHA, New York Heart Association; CABG, coronary artery bypass grafting; IQR, interquartile range; ICD, implantable cardioverterdefibrillator; $N / A$, not applicable.

(without transplantation) was significantly better in groups 2 and $3(P=.001)$, which had similar survival curves at 1 year (Figure 2). In patients with a preoperative ICD (group 1), 6 (20\%) of 30 patients died, and among the remaining cohorts (groups 2 and 3), 14 (8\%) of 187 patients died $(P=.03)$. The causes of death were available for $18(90 \%)$ of 20 patients and are shown in Table 2. Most patients died from progressive myocardial failure, and 2 patients died from malignancy. One sudden death occurred in group 1 . The survival between $\mathrm{EP}^{+}$and $\mathrm{EP}^{-}$patients was similar (Figure 3).

In patients who were discharged from the hospital with ICDs in situ, $6(20 \%)$ patients in group 1 and $9(12 \%)$ in group 2 had appropriate ICD therapies. Overall, the independent predictors of ICD therapy included preoperative left ventricular end-diastolic dimensions (relative risk, 2.92; 95\% confidence interval, 1.22-7.19; $P=.02$ ), left ventricular end-systolic dimensions (relative risk, $2.61 ; 95 \%$ confidence interval, 1.24-5.94; $P=.02$ ), LVEF (relative risk, $0.89 ; 95 \%$ confidence interval, $0.79-0.99 ; P=.03$ ), and concomitant CABG (relative risk, 0.51; protective; 95\% confidence interval, $0.30-0.91 ; P=.03$ ). Four patients, despite a negative EP study, received ICDs for secondary prevention. Of these, none received ICD therapy.

The 9 therapies delivered in group 2 included antitachycardia pacing in 3 and shocks in 6 . EP studies were highly sensitive in predicting ICD therapies- 8 of 9 therapies were delivered to patients with positive studies, whereas 1 patient who received multiple therapies had secondary prevention as the indication for ICD.

The median time to first ICD therapy was 43 days (Figure 4). Most (67\%) first therapies occurred within the first 63 days. In group 2, which had ICDs implanted after LVR, the first arrhythmia occurred 13 days after surgery, and two thirds of first events occurred within 63 days.

\section{Discussion}

This study demonstrates that patients remain at high and early risk for ventricular arrhythmias after LVR. Among 


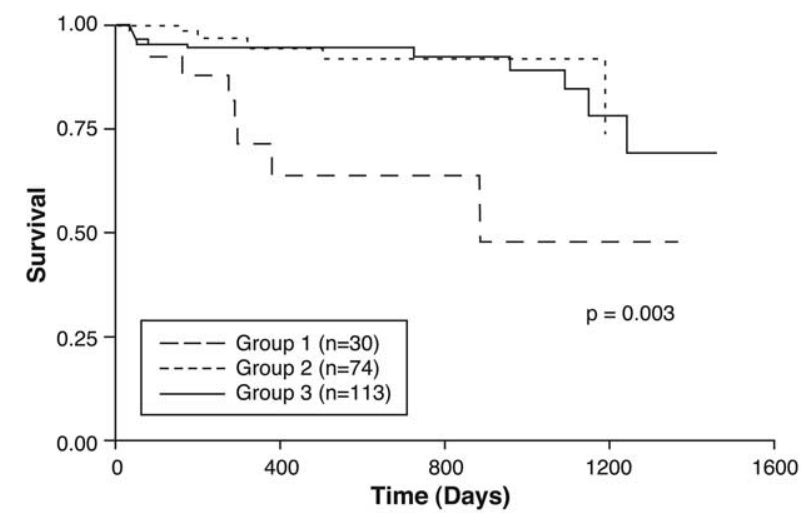

No. at risk

$\begin{array}{ll}\text { Group 1 } & 30 \\ \text { Group 2 } & 74\end{array}$

Group $3 \quad 113$

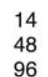

9

34
96

0

12

Figure 2. Kaplan-Meier survival curve according to groups (censored for cardiac transplantation).

patients who underwent EP studies, $42 \%$ tested positive for inducible sustained ventricular arrhythmias. In patients with ICDs, $15 \%$ had either sudden cardiac death or appropriate ICD shocks. ICD therapies tended to occur early, within the first 60 to 90 days. With the strategy of early EP study, ICD implantation, or both, the overall incidence of sudden death was less than $1 \%$.

The low incidence of sudden death is remarkable because these patients had very advanced heart failure due to ischemic cardiomyopathy, with a mean LVEF of $22 \%$. As such, they were at high risk for the development of ventricular arrhythmias. Most had limiting heart failure symptoms (68\% New York Heart Association class III/IV before surgery). Of interest, New York Heart Association class IV heart failure is usually considered a contraindication to ICD implantation. Furthermore, sudden death (assumed to be due to arrhythmia) occurred in 7 of 147 patients who underwent left ventricular aneurysm repair at single center and who were followed up for a median on 3.7 years. This rate indicates a very high propensity for lethal ventricular arrhythmias in this patient population. ${ }^{13}$ Of note, none of these patients received antiarrhythmic therapy.

At present, there are no recommendations regarding the use and timing of ICDs in patients undergoing LVR. Nearly all $(85 \%)$ patients had an LVEF of $30 \%$ or less and, as such, would fit MADIT II criteria for prophylactic ICD implantation for primary prevention of sudden cardiac death. ${ }^{10}$ However, patients were excluded from MADIT II if they were within 3 months of CABG. In the LVR population studied here, $67 \%$ of those who received ICD therapies had their first therapy within 90 days after LVR. Had we waited 3 months before implantation of an ICD, these patients may have been at a significant risk for potentially preventable sudden cardiac death. The opportunity to improve survival by the early application of EP testing, ICD implantation, or both in patients undergoing LVR is highly relevant, because performance of this procedure is growing. The strategy we used in group 2 of early ICD implantation or EP-guided ICD implantation seemed successful in view of the low incidence of sudden cardiac death observed in this group.

The effects of EP studies and ICD therapies have not been analyzed in other studies of LVR, including a large observational study (Surgical Anterior Ventricular Endocardial Restoration) of 439 patients undergoing LVR. In this study, which included 114 patients from our institution, survival was $84 \%$ at 18 months. ${ }^{14}$ It is of interest that the population presented in the current study had a lower LVEF (22\% vs $29 \%$ ) yet had a similar survival.

Whether EP risk stratification or ICD implantation without EP testing is warranted before discharge after LVR has been debated. In light of MADIT II, the utility of EP studies in patients with coronary disease and ejection fractions less than $30 \%$ has been questioned, because the benefit of ICD implantation occurs irrespective of EP testing. In the population presented here, however, negative EP studies were predictive of good 30-day survival. No patient with a negative EP study died within 30 days ( 2 died within 90 days). Of the 46 patients in group 3 who did not have an EP study, however, 8 died during follow-up (3 during the first 90 days and none before 30 days). Furthermore, delaying EP risk stratification or ICD implantation until 3 months after surgery could, arguably, have resulted in avoidable sudden cardiac deaths.

In this study, long-term survival in patients with positive EP studies was similar to that in patients with negative EP studies. This finding may reflect the protective effect of ICD implantation in patients with positive studies, thus indicating effective risk stratification and treatment for high-risk

TABLE 2. Causes of death after left ventricular reconstruction

\begin{tabular}{ccccccc}
\hline Group & $\begin{array}{c}\text { Total } \\
\text { deaths }\end{array}$ & $\begin{array}{c}\text { Sudden } \\
\text { death }\end{array}$ & $\begin{array}{c}\text { Cardiac } \\
\text { dysfunction* }\end{array}$ & Malignancy & $\begin{array}{c}\text { Respiratory } \\
\text { failure }\end{array}$ & Unknown \\
\hline 1 & 6 & 1 & 5 & 0 & 0 & 0 \\
2 & 3 & 0 & 2 & 0 & 1 & 0 \\
3 & 11 & 0 & 7 & 2 & 0 & 2 \\
\hline
\end{tabular}

*Cardiac dysfunction includes death from myocardial infarction, progressive heart failure, multiple organ failure, and other cardiac (unspecified) causes. 


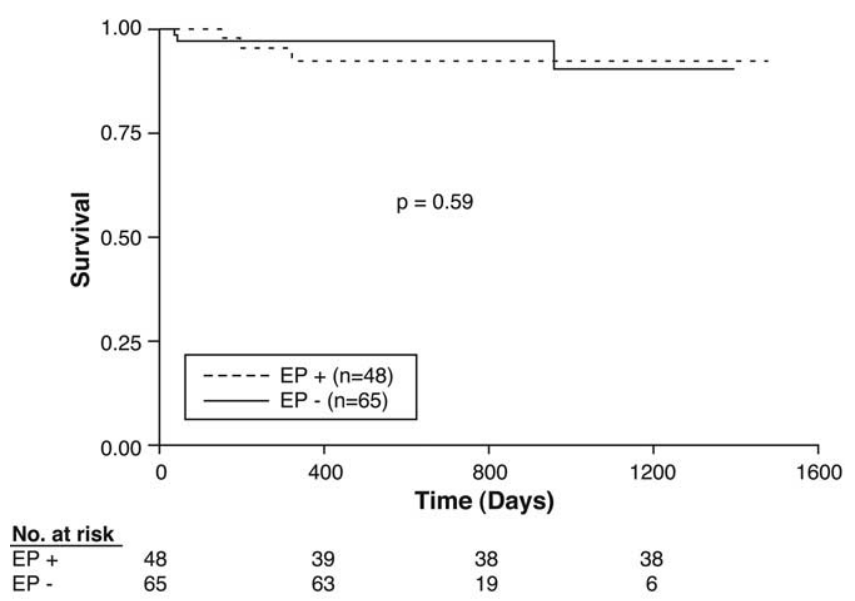

Figure 3. Kaplan-Meier survival curve (censored for cardiac transplantation) according to electrophysiologic (EP) results.

patients. Indeed, a positive EP study was predictive of 8 of 9 ICD therapies delivered in group 2. However, limitations in the ability of EP studies to stratify risk in this high-risk population cannot be completely excluded.

Group 1 in this study had the worst survival, despite the presence of preoperative ICDs. The poorer survival may have been related to the greater severity of their cardiomyopathy, as manifested by larger, less contractile ventricles and the presence of a preoperative ICD (a marker for a history of or risk for life-threatening cardiac arrhythmias). Because most deaths occurred as a result of progressive myocardial failure, rather than sudden arrhythmic events, these predictors may be markers of a residual propensity to develop progressive heart failure. Whether these patients should undergo LVR is another issue to be addressed by the STICH trial.

The question of whether LVR increases or decreases the likelihood of ventricular arrhythmias is complex. Removal of myocardial scarring may protect from ventricular arrhythmias. However, the resultant ventriculectomy scar may be proarrhythmic. In Dor and colleagues' ${ }^{15}$ original description of the procedure, recurrent ventricular arrhythmia was the indication for operation in $8 \%$ of patients. Postoperative follow-up regarding ventricular arrhythmia events in this series was unclear. In a larger later series, however, Dor ${ }^{16}$ (who routinely uses cryoablation and endocardial resection) reported an $8 \%$ incidence of inducible ventricular tachycardia after surgery. In their initial study on the outcome of LVR, the Reconstructive Endoventricular Original Radius Elliptical Shape to the Left Ventricle group reported arrhythmic deaths in 4 of 8 late deaths, which occurred in 207 patients who survived to discharge from the hospital after LVR. ${ }^{17}$ In a Japanese study of LVR in patients with only akinetic left ventricular scars, $3(6 \%)$ of 47 died from

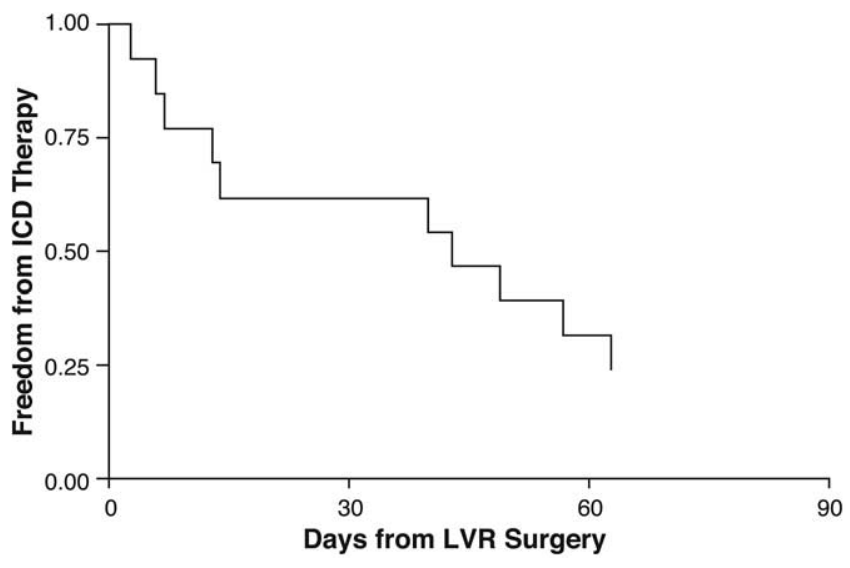

Figure 4. Time to first therapy (within 90 days of LVR surgery) in patients with ICD firings ( $\mathrm{n}=15$ ).

arrhythmic deaths after discharge. ${ }^{18}$ Mickleborough $^{19}$ reported a very low incidence of ventricular arrhythmias after left ventricular aneurysmectomy and a 79\% 5-year survival. By comparison, in the current study, the rate of positive EP studies was $48(42 \%)$ of 113 , and the risk of arrhythmic events (ICD therapies plus sudden death mortality) in groups 1 and 2 was $16(15 \%)$ of 104 . Nevertheless, the overall incidence of sudden death was $1(0.5 \%)$ of 217 by using the current strategy of early risk stratification and/or ICD implantation when feasible. These issues will be important to analyze in the ongoing STICH trial, a large randomized prospective study comparing medical versus surgical management (with or without LVR) of patients with ischemic cardiomyopathy. ${ }^{1}$

Patients undergoing LVR remain at risk for life-threatening arrhythmias, and EP-guided ICD implantation may result in improved short-term and intermediate-term survival. Predictors of ICD therapies included larger left ventricular dimensions, lower ejection fraction, and lack of concomitant revascularization, thus suggesting that the degree of myocardial dysfunction may remain a prime indicator of residual risk for ventricular arrhythmias. Because of the early risk of arrhythmias in patients after LVR, we suggest that either early ICD implantation or predischarge EP study for risk stratification is indicated.

This was an observational study in patients who were selected as suitable for LVR. It was nonrandomized, and EP testing was performed at the discretion of the treating physician. The number of outcome events was small and did not allow for multivariable modeling. Nevertheless, this study remains among the largest published series regarding the outcome of the LVR procedure.In conclusion, patients undergoing LVR remain at significant risk for ventricular arrhythmias. The time to the first arrhythmic event is short. Two thirds of first arrhythmic events occurred within the 
first 90 days after surgery. Many patients undergoing LVR meet MADIT II criteria for prophylactic ICD implantation. The data presented here support the use of early ICD implantation or EP-guided ICD therapy before hospital discharge.

\section{References}

1. Jones RH. Is it time for a randomized trial of surgical treatment of ischemic heart failure? J Am Coll Cardiol. 2001;37:1210-3.

2. Moss AJ, Hall WJ, Cannom DS, Daubert JP, Higgins SL, Klein H, et al. Improved survival with an implanted defibrillator in patients with coronary disease at high risk for ventricular arrhythmia. Multicenter Automatic Defibrillator Implantation Trial Investigators. $N$ Engl J Med. 1996;335:1933-40.

3. Moss AJ, Zareba W, Hall WJ, et al. Prophylactic implantation of a defibrillator in patients with myocardial infarction and reduced ejection fraction. N Engl J Med. 2002;346:877-83.

4. Centers for Medicare \& Medicaid. Decision memo for implantable cardioverter defibrillators (ICDs) (CAG-00157N). Available at: http:// www.cms.hhs.gov/mcd/viewdecisionmemo.asp?id=39. Accessed June 20, 2004.

5. Mahon NG, O’Neill JO, Young JB, Bennett R, Hoercher K, Banbury MK, et al. Contemporary outcomes of outpatients referred for cardiac transplantation evaluation to a tertiary heart failure center: impact of surgical alternatives. J Card Fail. 2004;10:273-8.

6. O'Neill JO, Starling RC. Surgical remodeling in ischemic cardiomyopathy. Curr Treat Options Cardiovasc Med. 2003;5:311-9.

7. Caldeira C, McCarthy PM. A simple method of left ventricular reconstruction without patch for ischemic cardiomyopathy. Ann Thorac Surg. 2001;72:2148-9.

8. Buxton AE, Lee KL, DiCarlo L, Gold MR, Greer GS, Prystowsky EN, et al. Electrophysiologic testing to identify patients with coronary artery disease who are at risk for sudden death. $N$ Engl J Med. 2000;342:1937-45.

9. Bigger JT Jr, Whang W, Rottman JN, Kleiger RE, Gottlieb CD, Namerow PB, et al. Mechanisms of death in the CABG patch trial: a randomized trial of implantable cardiac defibrillator prophylaxis in patients at high risk of death after coronary artery bypass graft surgery. Circulation. 1999;99:1416-21.

10. Coats AJ. MADIT II, the Multi-center Autonomic Defibrillator Implantation Trial II stopped early for mortality reduction, has ICD therapy earned its evidence-based credentials? Int J Cardiol. 2002;82: $1-5$.

11. Lee KL, Hafley G, Fisher JD, Gold MR, Prystowsky EN, Talajic M, et al. Effect of implantable defibrillators on arrhythmic events and mortality in the multicenter unsustained tachycardia trial. Circulation. 2002;106:233-8.

12. Hummel JD, Strickberger SA, Daoud E, Niebauer M, Bakr O, Man $\mathrm{KC}$, et al. Results and efficiency of programmed ventricular stimulation with four extrastimuli compared with one, two, and three extrastimuli. Circulation. 1994;90:2827-32.

13. Matthias Bechtel JF, Tolg R, Graf B, Richardt G, Noetzold A, Kraatz EG, et al. High incidence of sudden death late after anterior LVaneurysm repair. Eur J Cardiothorac Surg. 2004;25:807-11.

14. Athanasuleas CL, Stanley AW Jr, Buckberg GD, Dor V, DiDonato M, Blackstone EH. Surgical anterior ventricular endocardial restoration (SAVER) in the dilated remodeled ventricle after anterior myocardial infarction. RESTORE group. Reconstructive Endoventricular Surgery, returning Torsion Original Radius Elliptical Shape to the LV. J Am Coll Cardiol. 2001;37:1199-209.

15. Dor V, Saab M, Coste P, Kornaszewska M, Montiglio F. Left ventricular aneurysm: a new surgical approach. Thorac Cardiovasc Surg. 1989;37:11-9.

16. Dor V. The treatment of refractory ischemic ventricular tachycardia by endoventricular patch plasty reconstruction of the left ventricle. Semin Thorac Cardiovasc Surg. 1997;9:146-55.

17. Di Donato M, Toso A, Maioli M, Sabatier M, Stanley AW Jr, Dor V. Intermediate survival and predictors of death after surgical ventricular restoration. Semin Thorac Cardiovasc Surg. 2001;13:468-75.

18. Suma H, Isomura T, Horii T, Hisatomi K. Left ventriculoplasty for ischemic cardiomyopathy. Eur J Cardiothorac Surg. 2001;20:319-23.

19. Mickleborough LL. Left ventricular reconstruction for ischemic cardiomyopathy. Semin Thorac Cardiovasc Surg. 2002;14:144-9.

\section{The Journal of Thoracic and Cardiovascular Surgery Conflict of Interest Policy}

To assure fairness to authors submitting work for consideration in The Journal of Thoracic and Cardiovascular Surgery, a mechanism exists for managing conflicts of interest. The editor and each of the section editors complete a "Conflict of Interest" form that identifies any and all relationships with commercial and other academic entities. When the editor has a potential conflict because of a relationship with another entity or author, the editor appoints an alternate editor from among the section editors or editorial board members who assumes the entire responsibility for final decisions on the manuscript in question. The editor does not read the reviews that are submitted nor engage in discussing the manuscript prior to the final decision. When the conflict of interest involves a section editor, a "guest section editor" is appointed who fills the role normally played by the conflicted section editor. All members of the editorial board and reviewers are asked to indicate any conflict of interest when they agree to review a manuscript. 